\title{
Could Daily Monitoring of Fibrin Related Markers Help Suspect a Thrombotic Event in COVID-19 Patients? A Prospective Pilot Study
}

\author{
Michael Hardy $1,2(0)$ \\ Isabelle Michaux ${ }^{3}$ \\ Alain Dive ${ }^{3}$ \\ 1 Université catholique de Louvain, CHU UCL Namur, Namur \\ Thrombosis and Hemostasis Center (NTHC), Namur Research \\ Institute for Life Sciences (NARILIS), Hematology Laboratory, Yvoir, \\ Belgium \\ 2 Université catholique de Louvain, CHU UCL Namur, Namur \\ Thrombosis and Hemostasis Center (NTHC), Namur Research \\ Institute for Life Sciences (NARILIS), Anesthesiology Department, \\ Yvoir, Belgium \\ ${ }^{3}$ Université catholique de Louvain, CHU UCL Namur, Intensive Care \\ Department, Yvoir, Belgium \\ ${ }^{4}$ Division of Angiology and Hemostasis - Geneva Platelet Group, \\ Departement of Medicine, Université de Genève et Hôpitaux \\ Universitaires de Genève, Geneva, Switzerland
}

TH Open 2021;5:e152-e154.

Coronavirus disease 2019 (COVID-19) is associated with a high incidence of thrombotic events (TE). ${ }^{1}$ Prompt recognition of thrombosis in COVID-19 patients is highly relevant. Signs heralding a TE can be very difficult to detect however, particularly in the intensive care setting. What a proper clinical suspicion of an acute VTE is in that setting remains elusive and documenting a pulmonary event can be a huge challenge for such severely affected, unstable patients. Therefore, the value of laboratory markers indicating fibrin formation in vivo in increasing the suspicion of an on-going TE deserves to be studied.

D-dimers are cross-linked fibrin degradation (plasmin) products containing the D-dimer motif. Their plasma levels indicate the risk for TE in COVID-19 patients. ${ }^{2-6}$ Assays for D-dimers are widely and readily available, around the clock. Rises during hospital stay could point to the occurrence of TE. ${ }^{6}$ However, D-dimers plasma levels are elevated in COVID-19 patients already on admission ${ }^{6,7}$; therefore finding a clinically useful threshold to alert of a possible on-going TE is not easy. Fibrin monomers (FM) are other 'fibrin-related markers' (FRM), which differ from D-dimers, their formation not depending on fibrinolysis, which is impaired in COVID-19 patients. ${ }^{8,9}$ As D-dimers, FM are easily measured in citrated

\footnotetext{
$\bar{*}$ Equally contributed.
}

\author{
Thomas Lecompte ${ }^{4, *}$ François Mullier ${ }^{1, *}$
}

Address for correspondence François Mullier, Professor, Hematology Laboratory, Namur Thrombosis and Hemostasis Center, Université Catholique de Louvain, CHU UCL Namur, Avenue G. Thérasse, 1, B-5530 Yvoir, Belgium (e-mail: francois.mullier@uclouvain.be).

samples and can rise in case of both arterial and venous thrombosis. ${ }^{10}$ To the best of our knowledge, there are few available data on FM in COVID-19 patients. ${ }^{11,12}$ We therefore undertook a prospective observational exploratory monocentre study to get some insight as to whether their serial monitoring might help suspect an on-going thrombosis.

Twenty-one consecutive patients admitted to the intensive care unit (ICU) of an academic hospital with reverse transcription-polymerase chain reaction confirmed COVID19 , all prophylactically administered with heparin, were included from March 27 to April 24, 2020. ${ }^{8}$ Enoxaparin was the preferred anticoagulant. Unfractionated heparin (UFH) was used in case of extracorporeal oxygen membrane oxygenation (ECMO), renal failure or high bleeding risk. FRM (D-dimers and FM in soluble fibrin complexes - Liatests, Stago; normal FM values $<6 \mu \mathrm{g} / \mathrm{mL}$; FM limit of quantification $5 \mu \mathrm{g} / \mathrm{mL}$, even though reported measures are sometimes lower ${ }^{13}$ ) were measured in double-centrifuged frozenthawed citrated plasma samples, daily prepared. $^{8}$ Deep vein thrombosis (DVT) was diagnosed by ultrasound examination when clinically suspected and systematically once a week. Pulmonary thrombosis - embolism was diagnosed when clinically suspected by computed tomography angiography or by cardiac echography according to patient's status.

\section{(c) 2021. The Author(s).}

This is an open access article published by Thieme under the terms of the Creative Commons Attribution License, permitting unrestricted use, distribution, and reproduction so long as the original work is properly cited. (https://creativecommons.org/licenses/by/4.0/)

Georg Thieme Verlag KG, Rüdigerstraße 14, 70469 Stuttgart, Germany 
A
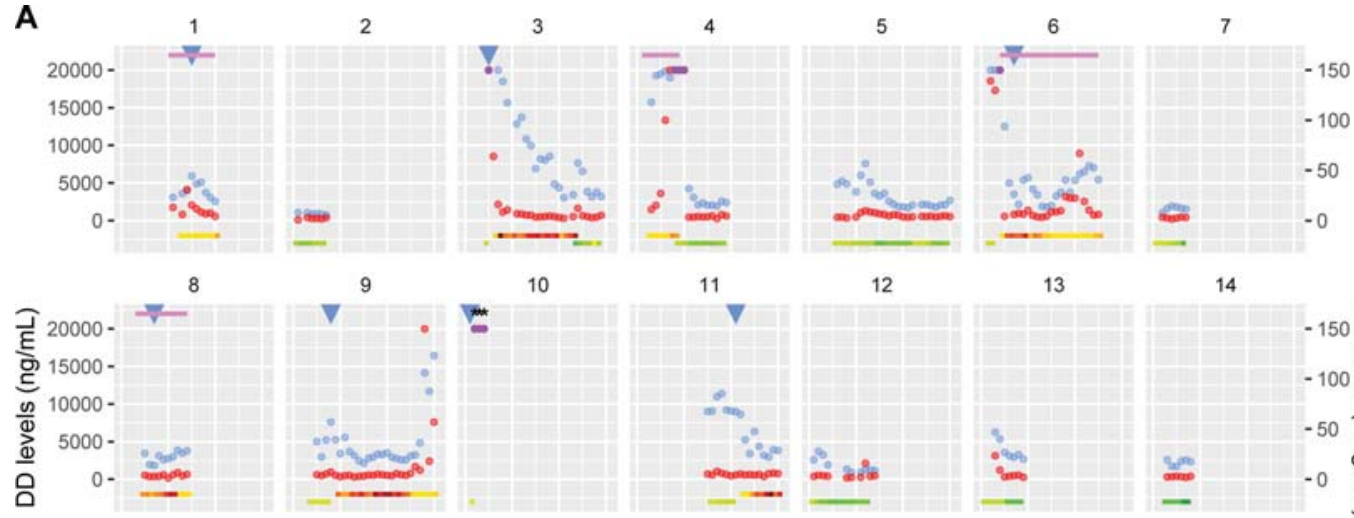

10
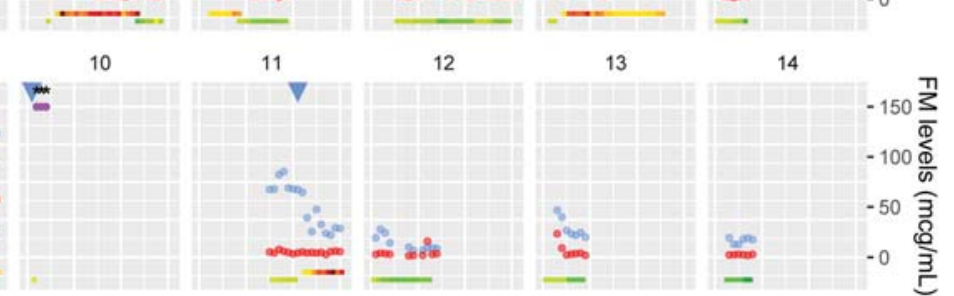

UFH anti-Xa activity $(\mathrm{IU} / \mathrm{mL})$
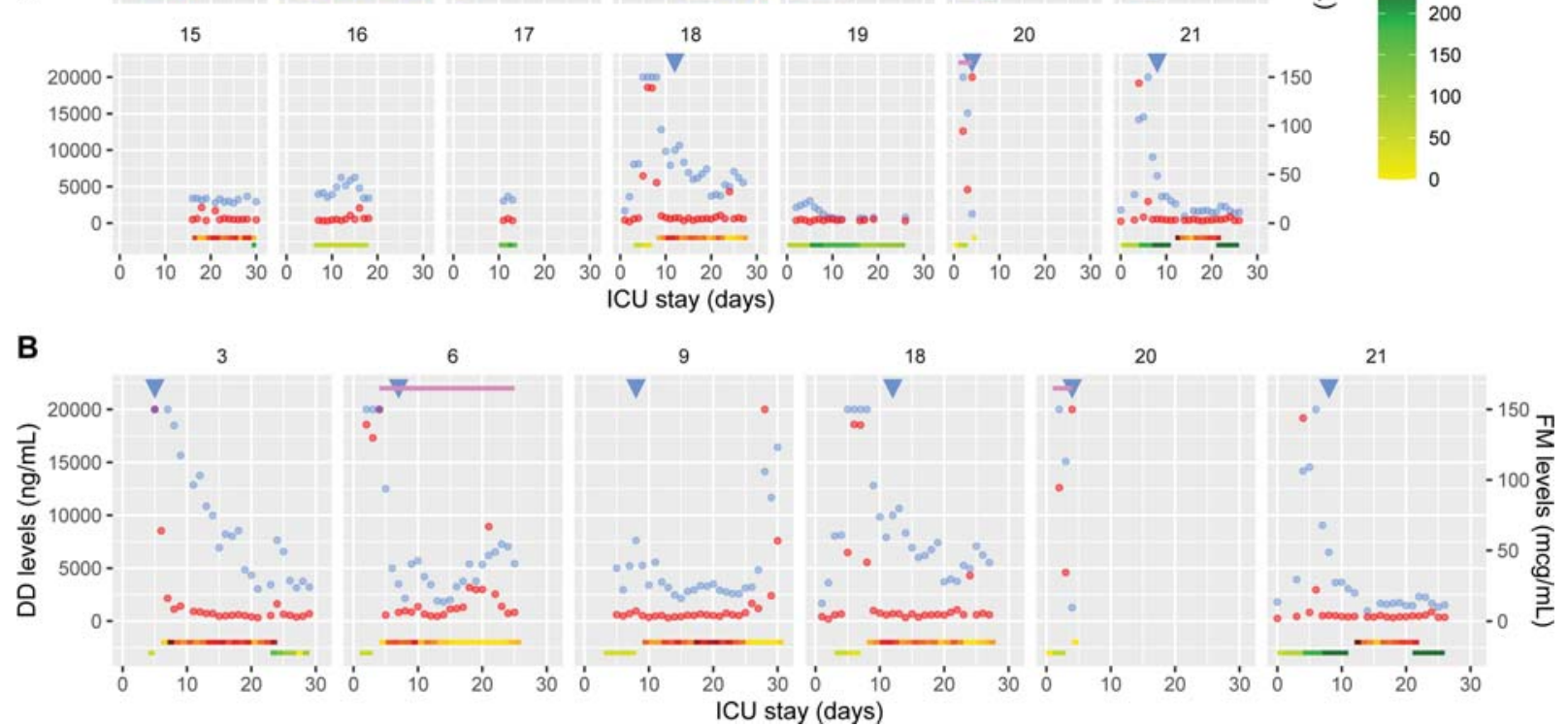

Fig. 1 Daily changes of D-dimers and fibrin monomers (FM) plasma levels during the intensive care unit stay for the 21 study patients (A) and for the six patients with a FM peak (B). The late FRM peak in patient 9 followed radioembolization of gluteal bleeding, which may explain the increase in FRM. ${ }^{19}$ D0 is the day of first ICU admission (11 patients were transferred from another ICU to ours). D-dimers levels are represented with blue dots and FM levels with red dots (purple dots correspond to superimposed values). Blue triangles correspond to days of thrombosis diagnosis and black stars to days when ISTH criteria for overt disseminated intravascular coagulopathy were met (either using D-dimers or FM as fibrin-related markers ${ }^{15}$ ). Upper pink lines represent the period of extracorporeal membrane oxygenation treatment. Daily LMWH (enoxaparin) received dose (green line) and achieved anti-Xa levels for UFH administration (red line) are represented in the bottom of the figure. UFH, unfractionated heparin; LMWH, low molecular weight heparin; DD, D-dimers; FM, fibrin monomers.

D-dimers changes along the ICU stay and their individual patterns were already reported. ${ }^{14}$

Ten patients were diagnosed with thrombosis, seven of them within eight days after ICU admission: five DVT (two symptomatic and three asymptomatic), two seemingly isolated PE, one patient with both, and two ischemic strokes. Five patients were under ECMO at some point of their ICU stay and one patient fulfilled ISTH overt disseminated intravascular coagulopathy criteria (DIC; both with D-dimers or FM as $\mathrm{FRM}^{15}$ ) while also suffering from ischemic stroke.

Results of both FRM along with main clinical features are represented over time in - Fig. 1 for each patient. The maximal FRM levels (medians) observed before censoring (TE, death, ICU discharge or end of study period) were higher in patients diagnosed with a TE than in patients who were not: 20,000 (IQR: 7,610-20,000) versus $3710 \mathrm{ng} / \mathrm{mL}(2,825-$ $6,265)$ for D-dimers ( $p=0.009$, Mann-Whitney U test); 139
(8-150) versus $10 \mu \mathrm{g} / \mathrm{mL}^{4-16}$ for FM ( $p=0.05$, MannWhitney $U$ test). We arbitrarily defined peak levels as follows: above $15,000 \mathrm{ng} / \mathrm{mL}$ or $100 \mu \mathrm{g} / \mathrm{mL}$ for D-dimers and FM respectively. Out of nine TE patients (one being excluded because no FRM levels were available before diagnosis of thrombosis), for five peaks preceded thrombosis diagnosis; and for the other 11 patients, ten were without; this held true for both FRM. Thus FRM peaks could help deciding which patients deserve imaging investigations. Additional studies should however be performed to confirm this hypothesis, to determine appropriate thresholds and to refine the respective role of each FRM.

In this regard, it is interesting to note that FM levels were often within the manufacturer's reference range (i.e., median proportion of ICU stay with FM below $6 \mu \mathrm{g} / \mathrm{mL}$ was $83 \%$ out of 299 measurements), in sharp contrast with very frequent high D-dimers. This argues against intravascular fibrin formation as the main contributor to D-dimers plasma levels, even in severe 
COVID-19 patients. The molecular mass of the former (basically one FM is associated with two fibrinogen molecules in a soluble complex: roughly a thousand $\mathrm{kDa}$ ) is such that what is circulating in blood most likely comes from the vasculature. ${ }^{16}$ By contrast, plasma D-dimers, which have lower masses than the former, could mainly come from extravascular deposits in the lungs. ${ }^{4,5} \mathrm{D}$-dimers levels would thus be more dependent on alveolar inflammation and damage - and thus on disease severity - than on intravascular fibrin formation..$^{17}$ Of note, FM levels were consistently high in two out of the five ECMO patients, which could come from fibrin deposition on the oxygenator membrane.

The important finding that FM plasma levels are often low is advantageous to capture an abrupt rise. Moreover, FM plasma levels decreased more rapidly after the peak than D-dimers. Indeed, FM peak levels were transient, perhaps specifically capturing intravascular fibrin formation, whereas D-dimers levels remained high for a more prolonged time-lapse. This could be explained by the shorter plasma half-life of FM and their production being less dependent on the fibrinolysis as compared with D-dimers. ${ }^{9}$

Our study has limitations: (i) few patients were studied (354 patient-days however, including 221 patient-days until censoring); (ii) regarding the time-course of FRM, some patients seemed to have been admitted at the onset of TE and a true FRM baseline level was therefore not available; (iii) precise determination of timing of TE is complicated; (iv) discrimination could be more difficult in case of DIC; only one patient fulfilled ISTH overt DIC criteria in the study, precluding any conclusion regarding this issue; (v) the course of FRM plasma levels is likely to be influenced by the anticoagulation regimen as well, further complicating the issue. ${ }^{18}$

To conclude, both FRM seem able to capture an on-going TE in most patients. An abrupt elevation should comfort in the clinical decision making to document TE and to consider therapeutic anticoagulation. Since D-dimers are always elevated in critical patients, sometimes markedly, FM plasma levels could be more striking warnings for an ongoing TE over a background of frequent normal levels and our data nominate it a candidate biomarker to investigate. As FM peaks are only transient, close monitoring would be required; whether it should be daily or otherwise deserves to be studied.

\section{Conflict of Interest}

The authors declare no competing interest related to this work.

\section{Acknowledgments}

The authors would like to thank Mrs Justine Baudar and Mrs Maité Guldenpfennig for performing the measurements.

This work was supported by the Belgian Fonds National de la Recherche Scientifique: 'Anticoagulation fibrinolysis COVID19' (reference: 40002796).

\section{References}

1 Klok FA, Kruip MJHA, van der Meer NJM, et al. Incidence of thrombotic complications in critically ill ICU patients with COVID-19. Thromb Res 2020;191:145-147

2 Choi JJ, Wehmeyer GT, Li HA, et al. D-dimer cut-off points and risk of venous thromboembolism in adult hospitalized patients with COVID-19. Thromb Res 2020;196:318-321

3 Mouhat B, Besutti M, Bouiller K, et al. Elevated D-dimers and lack of anticoagulation predict PE in severe COVID-19 patients. Eur Respir J 2020;56(04):2001811

4 Zermatten MG, Pantet O, Gomez F, et al; COVID-19 Interdisciplinary Collaboration - COVIDIC initiative. Utility of D-dimers and intermediate-dose prophylaxis for venous thromboembolism in critically ill patients with COVID-19. Thromb Res 2020;196:222-226

5 Dujardin RWG, Hilderink BN, Haksteen WE, et al. Biomarkers for the prediction of venous thromboembolism in critically ill COVID-19 patients. Thromb Res 2020;196:308-312

6 Naymagon L, Zubizarreta N, Feld J, et al. Admission D-dimer levels, D-dimer trends, and outcomes in COVID-19. Thromb Res 2020; 196:99-105

7 Huang C, Wang Y, Li X, et al. Clinical features of patients infected with 2019 novel coronavirus in Wuhan, China. Lancet 2020;395 (10223):497-506

8 Hardy M, Michaux I, Lessire S, et al. Prothrombotic disturbances of hemostasis of patients with severe COVID-19: A prospective longitudinal observational study. Thromb Res 2021;197:20-23

9 Dempfle CE. The use of soluble fibrin in evaluating the acute and chronic hypercoagulable state. Thromb Haemost 1999;82(02):673-683

10 Refaai MA, Riley P, Mardovina T, Bell PD. The Clinical Significance of Fibrin Monomers. Thromb Haemost 2018;118(11):1856-1866

11 Moosavi M, Wooten M, Goodman A, et al. Retrospective Analyses Associate Hemostasis Activation Biomarkers With Poor Outcomes in Patients With COVID-19. Am J Clin Pathol 2021;155(04): 498-505

12 Kumano O, Ieko M, Komiyama Y, et al. Characterization of fibrin/ fibrinogen degradation products reagents and their utility in critical care patients with enhanced fibrinolysis. Int J Lab Hematol 2020. Doi: $10.1111 /$ ijlh.13428

13 Toh JM, Ken-Dror G, Downey C, Abrams ST. The clinical utility of fibrin-related biomarkers in sepsis. Blood Coagul Fibrinolysis 2013;24(08):839-843

14 Hardy M, Michaux I, Lessire S, et al. Prothrombotic hemostasis disturbances in patients with severe COVID-19: Individual daily data. Data Brief 2020;33:106519

15 Suzuki K, Wada H, Imai H, Iba T, Thachil J, Toh CHSubcommittee on Disseminated Intravascular Coagulation. A re-evaluation of the Ddimer cut-off value for making a diagnosis according to the ISTH overt-DIC diagnostic criteria: communication from the SSC of the ISTH. J Thromb Haemost 2018;16(07):1442-1444

16 Dempfle CE, Wurst M, Smolinski M, et al. Use of soluble fibrin antigen instead of D-dimer as fibrin-related marker may enhance the prognostic power of the ISTH overt DIC score. Thromb Haemost 2004;91(04):812-818

17 Lippi G, Favaloro EJ. D-dimer is Associated with Severity of Coronavirus Disease 2019: A Pooled Analysis. Thromb Haemost 2020;120(05):876-878

18 Fassbender K, Dempfle CE, Mielke O, et al. Changes in coagulation and fibrinolysis markers in acute ischemic stroke treated with recombinant tissue plasminogen activator. Stroke 1999;30(10):2101-2104

19 Fernandez-Ros N, Iñarrairaegui M, Paramo JA, et al. Radioembolization of hepatocellular carcinoma activates liver regeneration, induces inflammation and endothelial stress and activates coagulation. Liver Int 2015;35(05):1590-1596 